\title{
DGCR8 Localizes to the Nucleus as well as Cytoplasmic Structures in Mammalian Spermatogenic Cells and Epididymal Sperm
}

\author{
Akane Nakano, Yuko Onohara, Sadaki Yokota, and Hideaki Fujita \\ Division of Functional Morphology, Faculty of Pharmaceutical Sciences, Nagasaki International University, Sasebo 859-3298, Japan \\ Correspondence should be addressed to Sadaki Yokota; syokota123@gmail.com
}

Received 4 September 2013; Revised 8 November 2013; Accepted 26 November 2013

Academic Editor: José R. Jaber

Copyright (c) 2013 Akane Nakano et al. This is an open access article distributed under the Creative Commons Attribution License, which permits unrestricted use, distribution, and reproduction in any medium, provided the original work is properly cited.

The localization of DGCR8 in spermatogenic cells and sperm from rat and mouse was studied by immunofluorescence and immunoelectron microscopy. Spermatogenic cells from these species yielded similar DGCR8 localization pattern. Immunofluorescence microscopy results showed that DGCR8 localized to both the cytoplasm and nucleus. In the cytoplasm, diffuse cytosolic and discrete granular staining was observed. Dual staining showed that DGCR8 colocalized to the granules with MAEL (a nuage marker). In the nucleus of spermatocytes, both the nucleoli and nucleoplasm were stained, whereas in the nucleus of early spermatids small spots were stained. In late spermatids, DGCR8 localized to the tip of their head and to small granules (neck granules) of the neck cytoplasm. The neck granules were also observed in the neck of epididymal sperm. Immunoelectron microscopy results showed that DGCR8 localized to nuage structures. Moreover, DGCR8 localized to nonnuage structures in late spermatids. DGCR8 also localized to the nucleolus and euchromatin in spermatocytes and round spermatids and to small granules in the nucleus of late spermatids. The results suggest that in spermatogenic cells DGCR8 localizes not only to the nuclei but also to the cytoplasmic structures such as nuage and nonnuage structures. Furthermore, DGCR8 seems to be imported into the egg with neck granules in sperm during fertilization.

\section{Introduction}

Spermatogenesis is a dynamic and highly complicated process that is composed of three phases based on functional aspects: (1) the proliferative phase in which spermatogonia undergo rapid successive cell divisions, (2) the meiotic phase in which recombination and segregation of chromosomes occur, and (3) the differentiation phase, named spermiogenesis, in which spermatids transform into spermatozoa which are specialized to carry haploid genome to the egg [1-3]. During these phases, numerous phase-specific and housekeeping genes are expressed and supply proteins are required for phase-specific functions and usual metabolism in testis [4].

RNA silencing pathway plays important roles in spermatogenesis [5-9]. A key step in this pathway is the processing of double-stranded (ds) RNAs into short RNA duplexes of characteristic size and structures, which is performed by Drosha and Dicer, proteins related to the RNase III family [10-13]. DGCR8 is a protein encoded to the DiGeorge syndrome critical region gene 8 and a subunit of microprocessor complex. It has a nuclear transport signal sequence and usually located in the nucleus, especially in the nucleolus together with nucleolin [14]. In the nucleus, DGCR8 binds Drosha to form the microprocessor complex that cleaves the primary microRNA (pri-miRNA) to the hairpin-shaped pre-microRNA (pre-miRNA). The resulting pre-miRNA is then exported to the cytoplasm and further processed to mature miRNA by Dicer, which is loaded together with Ago2 proteins into the RNA-induced silencing complex (RISC). The miRNA guides RISC to silence target mRNAs through mRNA cleavage, translation repression, or deadenylation [10-13]. It is known that processing body (P-body) or stress granules are cytoplasmic sites for RISC functions [15-19]. 
In male germ cells, similar compartments are nuage structures, including chromatoid bodies, which contain several components involved in miRNA pathway [20-22]. This idea is expanded to "germ granules" [23], which widely exist in germ cell line $[1,24]$. We have attempted to clarify the nuclear localization of DGCR8 in spermatogenic cells to know the localization of DGCR8 in differentiating spermatogenic cells. The results showed that DGCR 8 localized not only to the nucleus but also to diverse cytoplasmic structures. In this study, therefore, we investigate the precise localization of DGCR8 in spermatogenic cells and epididymal sperm of mammals using immunofluorescence (IF) and immunoelectron microscopy (IEM) techniques.

\section{Materials and Methods}

2.1. Animals. Male Wistar rats (weight $180-220 \mathrm{~g}$ ) and mice (25 g) were purchased from Kyudo Co. Ltd. (Tosu, Japan). All animals received standard diets and water ad libitum until use. Health epididymides of pig and horse were obtained from a slaughterhouse. Sperms were collected from epididymides of rat, mouse, pig, and horse. An experiment was performed in accordance with the guidance for Animal Experiments issued by the Nagasaki International University.

2.2. Antibodies and Related Probes. Rabbit anti-DGCR8 antibodies were purchased from ProteinTech (10996-1-AP, Chicago, IL, USA) and Abnova (MaxPab rabbit polyclonal antibody, D01, Taipei, Taiwan), respectively. Guinea pig antiMAEL antibody was used as described previously [25]. Alexa 568 or Alexa 488-conjugated goat anti-rabbit IgG or goat antiguinea pig IgG was obtained from Molecular Probes (Eugene, OR, USA). Horseradish peroxidase (HRP)-labeled swine antibody to rabbit IgG was purchased from DAKO Japan (Tokyo, Japan). Protein A-gold $15 \mathrm{~nm}$ probe was prepared as described previously [26].

2.3. Western Blotting. Testes of rat and mouse ( $250 \mathrm{mg}$ each) were homogenized in $5 \mathrm{~mL}$ of sample buffer for SDSPAGE. Homogenates were heated in boiling water for $5 \mathrm{~min}$ and centrifuged at $10,000 \times \mathrm{g}$ for $30 \mathrm{~min}$. The resulting supernatants were divided into $100 \mathrm{~mL}$ aliquots and stored at $-80^{\circ} \mathrm{C}$. Protein concentration of $5 \%$ homogenates $(\mathrm{w} / \mathrm{v})$ in PBS was determined by the bicinchoninic acid method (Pierce Chemical, Rockford, IL, USA) using bovine serum albumin as a standard. Fifty micrograms of each sample were analyzed by Western blotting. The apparent molecular mass of DGCR8 was estimated by a prestained protein maker (Nippon Genetics Europe, Düren, Germany).

2.4. Immunofluorescence Staining. Smear preparations of epididymal sperm from rat, mouse, pig, and horse, and frozen sections ( $6 \mu \mathrm{m}$ thick) of testes from rats and mice, were fixed in $4 \%$ paraformaldehyde $(\mathrm{w} / \mathrm{v})$ in $0.1 \mathrm{M}$ Hepes-KOH buffer ( $\mathrm{pH}$ 7.4) for $15 \mathrm{~min}$. After permeation treatment with $0.1 \%$ Triton X-100 (v/v) + 0.2\% Saponin (w/v), smear preparations and sections were incubated in $2 \%$ fish gelatin (w/v) in PBS for $30 \mathrm{~min}$ to block nonspecific adsorption of IgG, followed by overnight incubation with anti-DGCR8 antibodies from ProteinTech $(\times 100)$ or from Abnova $(\times 500$ by). After washing with PBS, the reacted IgG was visualized by Alexa 568 or Alexa 488-conjugated goat anti-rabbit IgG. For dual staining, sections were incubated with both rabbit anti-DGCR8 antibodies and affinity-purified guinea pig anti-MAEL $(1 \mu \mathrm{g} / \mathrm{mL})$ and each reacted IgG was visualized by Alexa 568-conjugated goat anti-rabbit IgG and Alexa 488-conjugated goat antiguinea pig IgG. For control, nonimmune sera were used instead of the specific primary antibodies. Nuclei were stained by $3 \mu \mathrm{M}$ DAPI (Hoechst, Tokyo, Japan) for $60 \mathrm{~min}$ at RT. The smear preparations and sections were examined with a Nikon Eclipse E600 fluorescence microscope (Tokyo, Japan). The images were merged with Adobe Photoshop 7.0 to determine whether each antigen colocalizes to the same area. The stage of the seminiferous cycle was determined from the localization of elongating and elongated spermatids with individual tubules as previously described [27].

2.5. Immunoelectron Microscopy. Rat and mouse testes were cut into small tissue blocks in fixative consisting of $4 \%$ paraformaldehyde $(\mathrm{w} / \mathrm{v}), 0.2 \%$ glutaraldehyde $(\mathrm{v} / \mathrm{v}), 0.02 \%$ $\mathrm{CaCl}_{2}(\mathrm{w} / \mathrm{v})$, and $0.1 \mathrm{M}$ Hepes- $\mathrm{KOH}(\mathrm{pH} 7.4)$ and tissue blocks were kept in the fixative for $1 \mathrm{~h}$ at $4^{\circ} \mathrm{C}$. Tissue blocks were dehydrated in ethanol and embedded in LR White at $-20^{\circ} \mathrm{C}$, followed by resin polymerization under UV light at $-20^{\circ} \mathrm{C}$. Thin sections were cut with a diamond knife equipped with a Reichert Ultracut $\mathrm{R}$, mounted onto nickel grids, and incubated with rabbit anti-DGCR8 antibodies from ProteinTech $(\times 100)$ or Abnova $(\times 500)$ overnight at $4^{\circ} \mathrm{C}$. For control experiments, nonimmune rabbit serum was used instead of the specific primary antibodies. The reacted IgG was visualized by $15 \mathrm{~nm}$ protein A-gold probe. Sections were contrasted with uranyl acetate and lead citrate, coated with evaporated carbon and examined with a Hitachi H7650 electron microscope (Tokyo, Japan) at an acceleration voltage of $80 \mathrm{kV}$. The stage of the seminiferous cycle and step of spermatids were determined as described by Russell et al. [27].

2.6. Routine Electron Microscopy. Testis tissue blocks from rats and mice were fixed in the fixative containing $4 \%$ paraformaldehyde (w/v), 1\% glutaraldehyde (v/v), $0.02 \%$ $\mathrm{CaCl}_{2}$ (w/v), and $0.05 \mathrm{M}$ Hepes-KOH (pH 7.4) overnight at $4^{\circ} \mathrm{C}$. After brief wash in PBS, testis tissue blocks were fixed in $1 \%$ reduced osmium tetroxide $(\mathrm{w} / \mathrm{v})$ for $1 \mathrm{~h}$, dehydrated, and embedded in Epon. Thin sections were contrasted with lead citrate and examined with electron microscope.

\section{Results}

3.1. Western Blotting. Two protein bands of 117 and $86 \mathrm{kDa}$ were observed in testis homogenates of rat and mouse with rabbit anti-DGCR8 antibody from ProteinTech (Figure 1). The same results were obtained with antibody from Abnova (data not shown). The band of $86 \mathrm{kDa}$ was consistent with the molecular weight of rat DGCR8 shown in SWISS-PROT data base. 


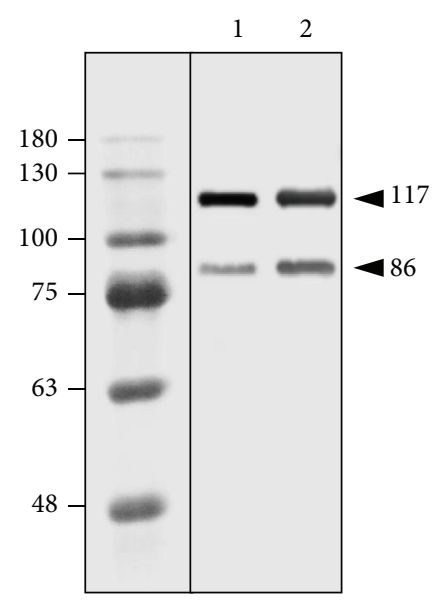

Figure 1: Western blotting of testicular extracts of rat and mouse. Lane 1: rat and lane 2: mouse. Numbers at left are molecular weight $(\mathrm{kDa})$ of protein markers and those at right are molecular weight of DGCR8 and its isomer estimated from the markers.

3.2. Immunofluorescence Staining. Immunofluorescence signals for DGCR8 were observed in three different compartments such as cytoplasmic granules, cytosol, and nuclei. Staining patterns changed during the stages cycle of seminiferous tubules. We observed carefully the staining pattern during spermatogenesis of rat testis. Cytoplasmic granular staining in spermatids was observed at steps 1-17 (Figures 2(a)2(c), 2(e), 2(g), and 2(h)). Weak cytoplasmic diffuse staining was observed in pachytene spermatocytes and spermatids throughout the stages (Figure 2). Nuclear diffuse staining was observed in spermatogonia and late spermatocytes (Figures 2(c), 2(d), and 2(e)), whereas nuclear granular staining was seen in round spermatids (Figures 2(b) and 2(c) arrowheads). In middle and late pachytene spermatocytes, spot staining was noted in the nuclei (Figure 2(d), arrowheads). In step 1719 spermatids, the tip and ventral surface of their head were strongly stained (Figures 2(b) and 2(c)). In step 13 spermatids, DGCR8 staining surrounded their head (Figure 2(e)). Furthermore, in the neck region of spermatids, small granules were stained (Figure 2(d), arrows). Staining patterns in each stage obtained with antibody from Abnova were quite similar to those with antibody from ProteinTech (Figures $2(\mathrm{~g})$ and 2(h)). In control, the staining described above was completely eliminated (Figure 2(f)).

Next we stained dually testis sections for DGCR8 and MAEL, a nuage protein marker, in order to clarify whether DGCR8-positive cytoplasmic granules are nuage. The results are shown in Figure 3. DGCR8-positive granules in round spermatids were stained also for MAEL, whereas granules in the cytoplasm of late spermatids were negative for MAEL (Figures 3(a)-3(c)). In late spermatocytes, MAEL-positive granules were not stained for DGCR8. In the cytoplasm of step 11-15 spermatids, granules stained for both DGCR8 and MAEL were observed (Figures 3(d)-3(f)). In some spermatocytes at stages V-VI, large spot within the nucleus was heavily stained for DGCR8 but not for MAEL (Figures $3(\mathrm{~d})-3(\mathrm{f}))$.

3.3. Immunoelectron Microscopy. Similar results were obtained with anti-DGCR8 antibodies from ProteinTech and Abnova.

3.3.1. Nuage Structures. We studied whether DGCR8 localized to nuage structures of rat and mouse, which previously we classified into five types [28]. Gold labeling was generally weaker than that for the other nuage proteins studied previously $[25,29-31]$ irregularly shaped perinuclear granules, intermitochondrial cement, and chromatoid bodies were labeled for DGCR8 (Figures 4(a)-4(f)). 70-90 nm particles and satellite bodies were not stained for DGCR8 (data not shown). No gold labeling in these structures was observed in control (Figure 4 insets).

3.3.2. Nonnuage Structures. Mitochondria-associated granules, which appeared in the elongated cytoplasmic lobe of late spermatids, were stained for DGCR8 (Figures 5(a) and 5 (b)). Ribosome aggregates, which emerged in the residual body of step 19 spermatids, were moderately labeled (Figures 5(c) and 5(d)). In addition, gold labeling for DGCR8 was observed in the small granules which were aggregated in the cytoplasm of step 17 spermatids and in the residual body (Figures 5(e) and 5(f)). The other nonnuage structures such as granulated bodies and reticulated bodies were negative for DGCR8 (data not shown). No gold labeling was seen in IEM control sections (Figure 5 Insets).

3.3.3. Nucleoplasm. The nucleoplasm of pachytene spermatocytes was moderately labeled but that of spermatids very weak or not. In spermatocytes, especially in late spermatocytes, most of the labeling was associated with euchromatin (Figure 6(a)). In step 6-7 spermatids, small dense particles, which aggregated to form a cluster, were labeled for DGCR8, whereas other nucleoplasm area was not labeled (Figure 6(b)). The nucleoplasm of step 10-11 spermatids was not stained excepting aggregated small dense granules (Figure 6(c)). No gold labeling was observed in IEM control sections (data not shown).

3.3.4. Nucleolus. Gold labeling for DGCR8 in the nucleolus was weaker in early to middle pachytene spermatocytes and became remarkable after stage IX, in which the nucleolus frequently came into close contact with XY body. In the nucleolus, DGCR8 mainly localized to the granular component surrounding the fibrillar center to which no DGCR8 localized (Figure 7(a)). Dense fibrillar component was also negative for DGCR8 (Figure 7(a)). In the nucleolus closely associated with XY body, DGCR8 was detected in both the granular component and in the fibrillar center (Figure 7(b)), whereas the dense fibrillar component was not labeled. The $\mathrm{XY}$ body was labeled very weak or not (Figure $7(\mathrm{~b})$ ). No gold labeling was observed in IEM control sections (data not shown). 


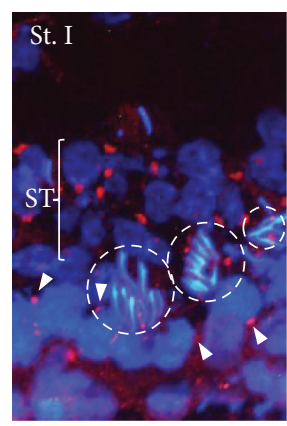

(a)

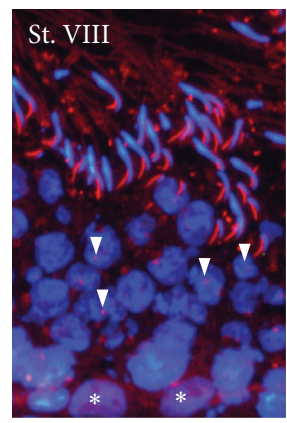

(c)

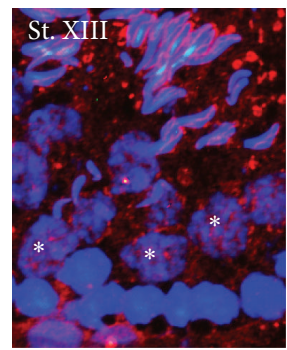

(e)

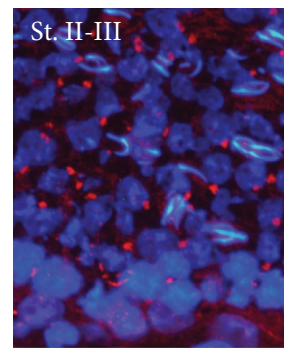

(g)

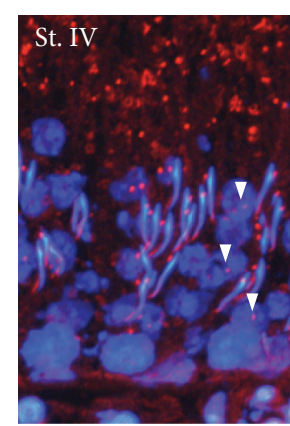

(b)

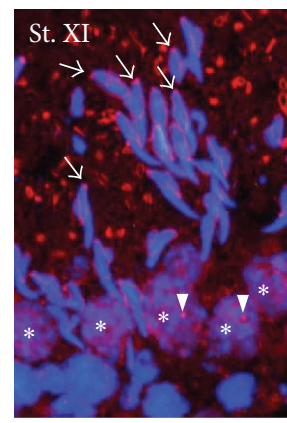

(d)

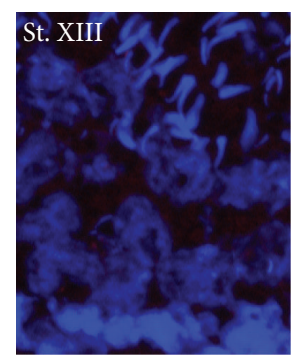

(f)

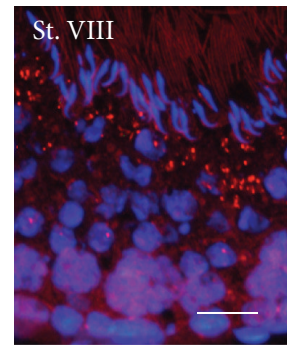

(h)

FIGURE 2: Stage-specific localization of DGCR8 during the spermatogenic cycle in the rat testis. Sections are stained DGCR8 (red) and DNA (bright blue). (a) Stage I. Seminiferous tubule is shown. In the perinuclear region of pachytene spermatocytes, granular staining is seen (arrowheads). In the cytoplasm of round spermatids (ST), granules are stained. Around the head of step 15 spermatids (circles), positive staining is observed. (b) Stage IV. In the nuclei of round spermatids, small spot is stained (arrowheads). In the rostral and caudal ends of spermatid head, wedge shape and oval staining are observed, respectively. Many granules in the cytoplasm of step 17 spermatids are stained. (c) Stage VIII. The nuclei $\left(^{*}\right)$ of spermatogonia are diffusely stained. Small spots in the nuclei of step 8 spermatids are stained (arrowheads), Wedge shape staining is seen at the tip of step 19 spermatid head. (d) Stage XI. The nuclei $\left(^{*}\right)$ of spermatocytes are diffusely stained, in which strongly stained spots are also seen (arrowheads). The heads of step 11 spermatids are surrounded by a stained line and small granules at the caudal end of the heads are stained (arrows). Many granules in the cytoplasm are stained. (e) Stage XIII. Patchy staining in the nuclei $\left({ }^{*}\right)$ and granular staining in the cytoplasm are noted in pachytene spermatocytes. Around the nuclei of step 13 spermatids, strong staining is seen and many granules in their cytoplasm are also positive for DGCR8. (f) Stage XIII. Control section. No red staining is observed. (g) and (h) Sections stained with anti-DGCR8 antibody from Abnova. Staining pattern is quite similar to that shown in (a) and (c). Bar $=10 \mu \mathrm{m}$. 


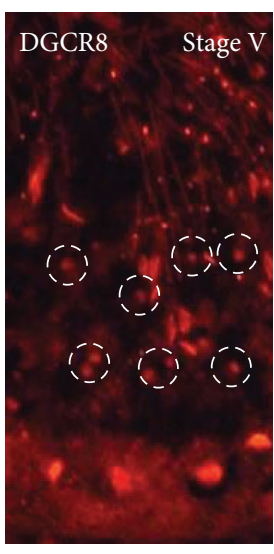

(a)

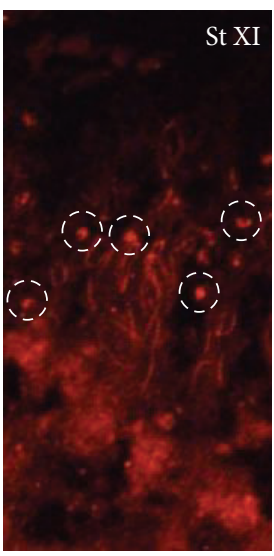

(d)

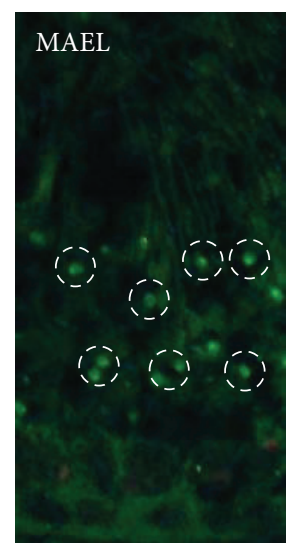

(b)

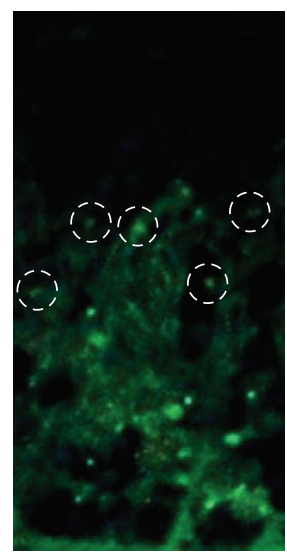

(e)

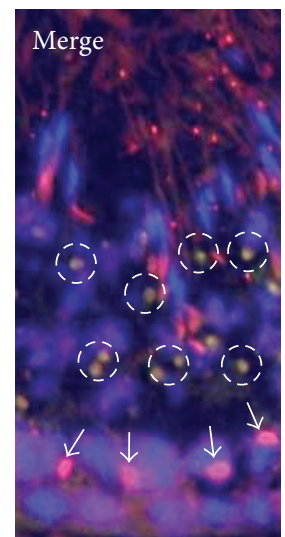

(c)

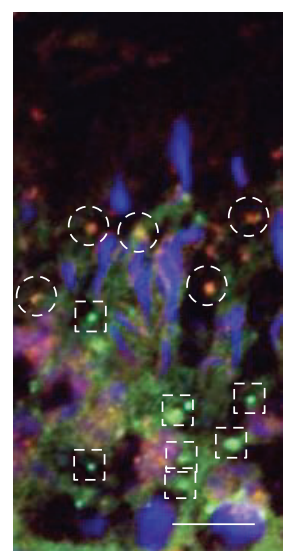

(f)

Figure 3: Dual staining of DGCR8 (red) and MAEL (green) in rat seminiferous tubules. (a, b, and c) Stage V. Relatively large spots in the nucleus of pachytene spermatocytes are stained only for DGCR8 (arrows). Granules in round spermatids are positive for both proteins (circles). Granules in step 17 spermatids are stained only for GDCR8. (d, e, and f) Stage XI. Granules in spermatocytes are stained only for MAEL (squares), whereas granules in step 11 spermatids are positive for both proteins (circles). Bar $=10 \mu \mathrm{m}$.

3.3.5. Dense Material in the Cytoplasm of Sertoli Cell Ectoplasmic Specialization. Ectoplasmic specializations are the complex cytoskeletal structure composed of the Sertoli cell plasma membrane closely contacted with spermatid head, the endoplasmic reticulum (ER) of this cell, and actin bundles between the plasma membrane and the ER [32]. DGCR8 localized to dense material located between the plasma membrane and the ER (Figure 8(a)).

3.3.6. Small Granule in the Neck Cytoplasm of Late Spermatids. This granule consisted of dense material, appeared in spermatids after step 9, and could be observed also in the epididymal sperm. It existed solitarily or attached to the neck piece of the flagellum. We named it neck granules. DGCR8 localized to the neck granules (Figures $8(\mathrm{~b})$ and $8(\mathrm{c})$ ). Although the electron density of cross-sectioned annulus was very similar to that of the neck granules, no DCGR8 was detected in it (Figures 8(b) and 8(c)). No gold signals were seen in IEM control section (Figure $8(d)$ ). The neck granules were evidently confirmed in routine electron microscopy (Figure 8(e)).

3.3.7. Neck Granules in Epididymal Spermatozoa. Next, we confirmed whether the neck granule existed in the neck cytoplasm of epididymal sperm from several mammals. Neck granules in sperm from rat, mouse, pig, and horse were stained for DGCR8 (Figures 9(a), 9(c), 9(d), and 9(e)). In rat and mouse, the number of stained granule was one or two, whereas in pig and horse, more than two granules were frequently stained (Figures 9(d) and 9(e)). No granules were stained in IF control preparation (Figure 9(b)).

\section{Discussion}

4.1. Antibodies. Rabbit anti-DGCR8 antibody from ProteinTech developed two protein bands in Western blotting. The same results were obtained with anti-DGCR8 antibody from Abnova. The molecular weight of the faster migrated band was $86 \mathrm{kDa}$, which was consistent with the molecular 


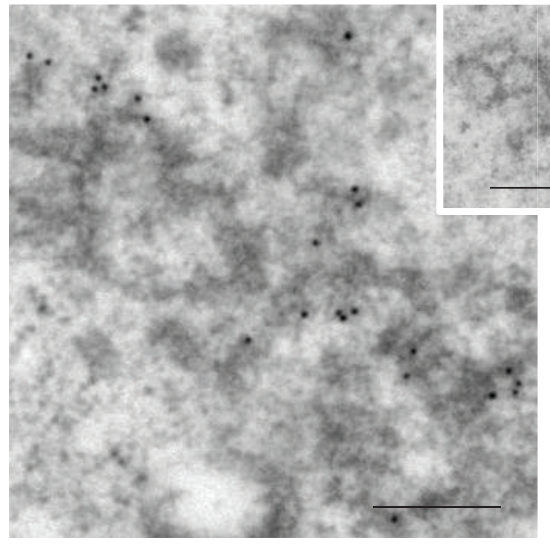

(a)

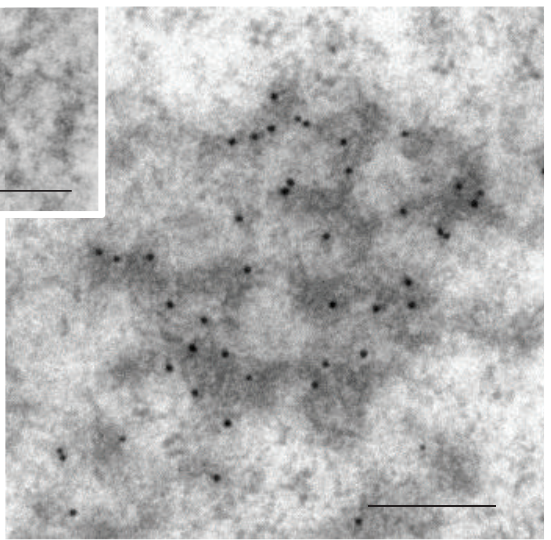

(b)

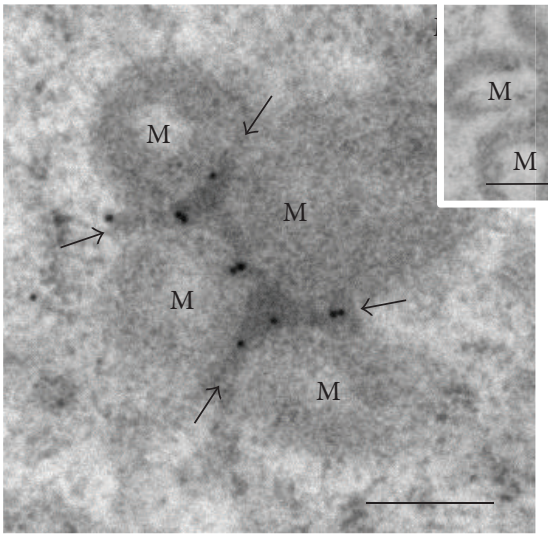

(c)

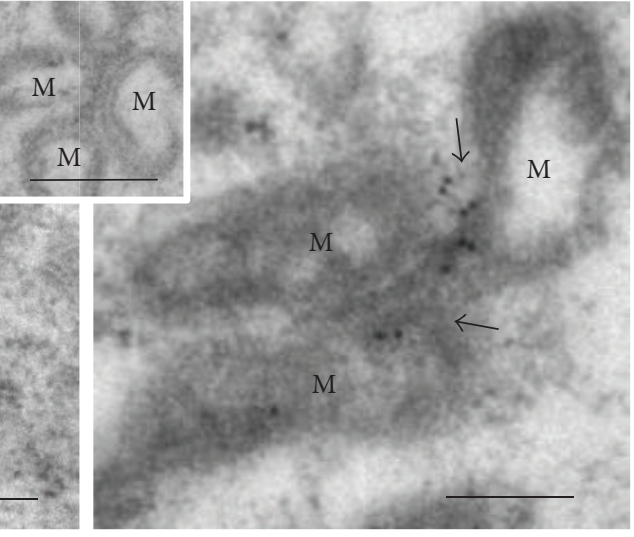

(d)

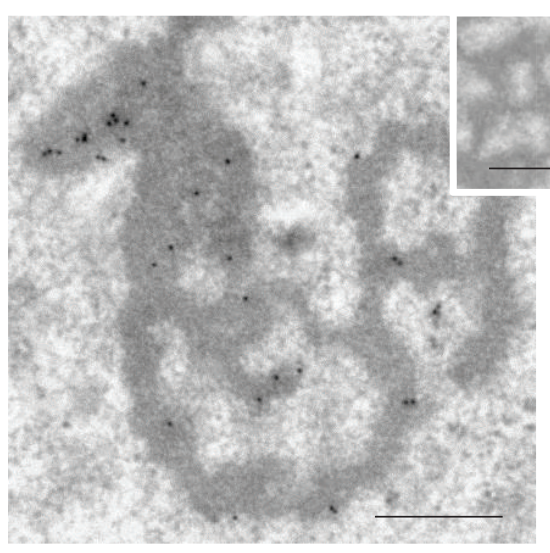

(e)

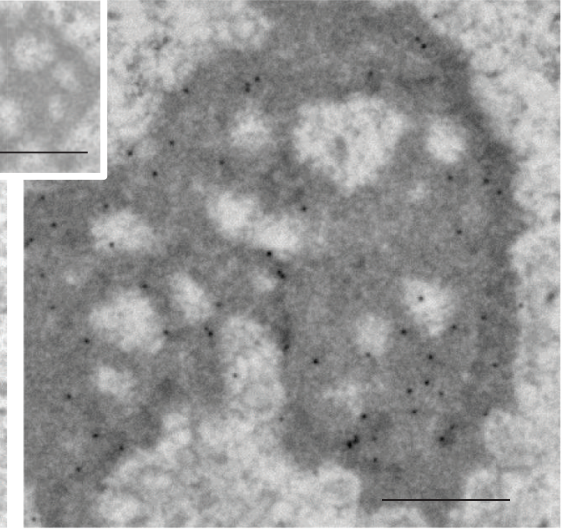

(f)

FIGURE 4: IEM localization of DGCR8 in nuage structures with two different antibodies. (a, c, and e) Staining with antibody from ProteinTech. (b, d, and f) Staining with antibody from Abnova. (a) and (b) Irregularly shaped perinuclear granules in mouse pachytene spermatocytes. Gold particles are observed on electron dense material. (c) and (d) Intermitochondrial cement in rat pachytene spermatocytes. Gold signals are seen on the dense material among the mitochondria (M) (arrows). (e) and (f) Chromatoid bodies in rat round spermatids. Gold particles are associated with dense material. No gold labeling is noted in IEM control (insets, all sections from rat). Bar $=0.5 \mu \mathrm{m}$.

weight estimated from amino acid sequence of DGCR8 cited in SWISS-PROT database. The molecular weight of the slowly migrated band was estimated to be $117 \mathrm{kDa}$. It has been reported that the nuclear localization sequence (NLS) of DGCR8 is 1-275 amino acid sequence [33] and later confined to be 256-289 sequence [14]. According to manufacturer datasheet, the protein with $117 \mathrm{kDa}(120 \mathrm{kDa}$ by manufacturer) is an isoform of DGCR8 but not well characterized so far. In the present study, these protein bands were also observed in Western blotting with anti-DGCR8 antibody 


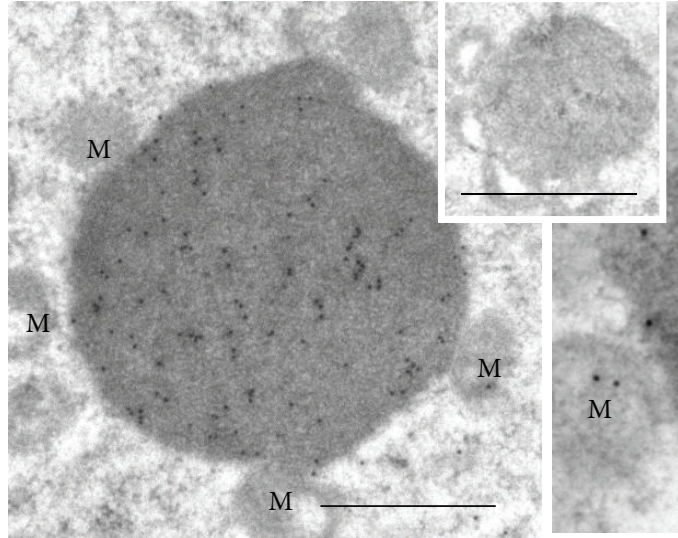

(a)

(c)

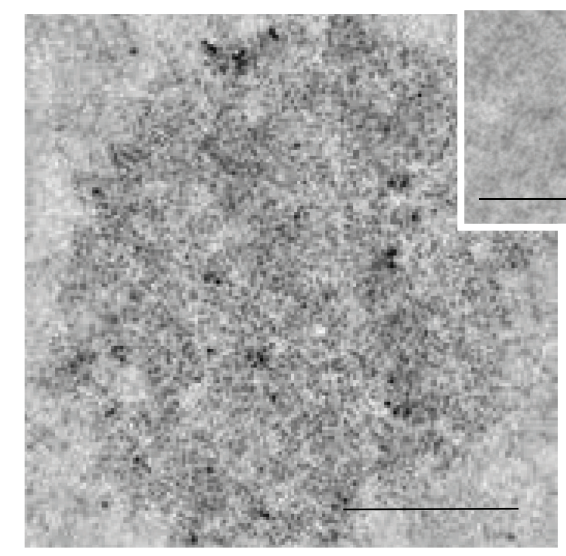

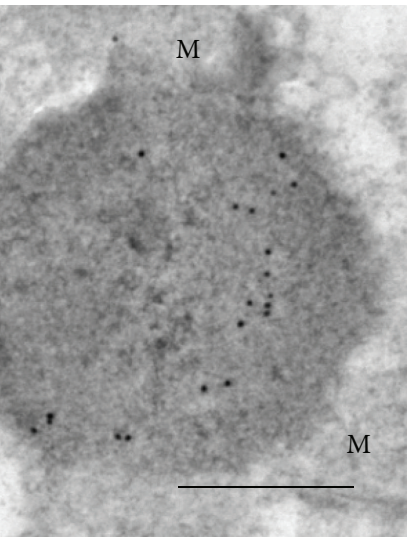

(b)

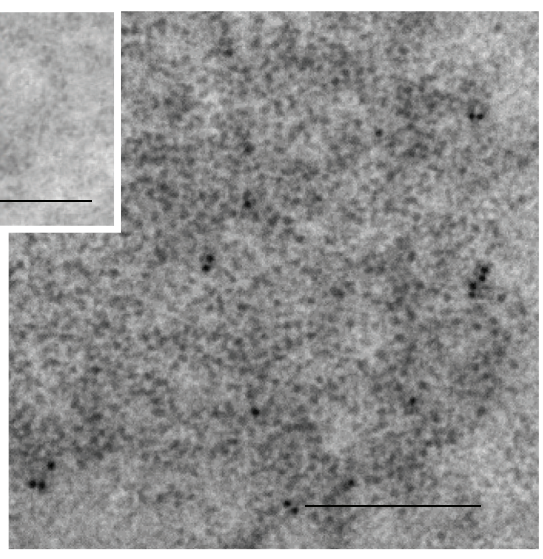

(d)

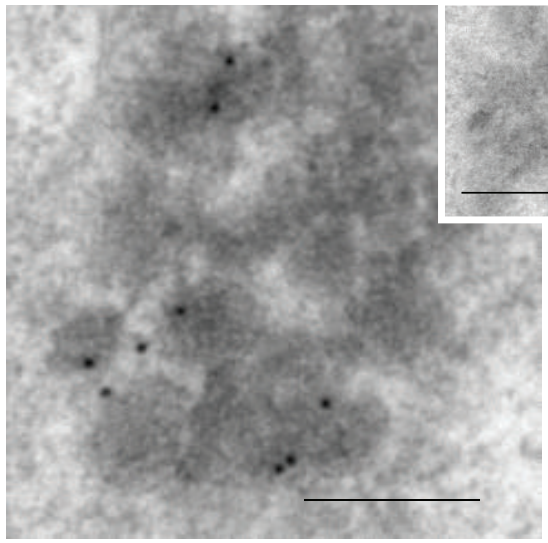

(e)

FIGURE 5: IEM localization of DGCR8 in nonnuage structures with two different antibodies. (a, c, and e) Sections from rat and staining with antibody from ProteinTech. (b, d, and f) Sections from mouse and staining with antibody from Abnova. (a and b) Mitochondria-associated granules. DGCR8 staining is present on fine granular dense material. M: mitochondria. (c and d) Ribosome aggregates. Gold particles are observed in the aggregate. (e and f) Small dense granules in residual body. Gold labeling is seen on the granules. No gold labeling is noted in IEM control (insets, all sections from rat). Bar $=1 \mu \mathrm{m}$ for ( $\mathrm{a}$ and $\mathrm{b}$ ) (inset) and $0.5 \mu \mathrm{m}$ for others.

from Abnova. Therefore, it is likely that $117 \mathrm{k}$ Da protein is a modified larger isoform of $86 \mathrm{kDa}$ protein, which has DGCR8 epitope(s). If so, both 86 and $117 \mathrm{kDa}$ proteins have the NLS and are able to localize to the nucleus. However, it is not clear which proteins localize to the cytoplasmic structures such as nuage and other structures investigated in this study.
4.2. Nuclear Localization of DGCR8 in Spermatogenic Cells. We showed that in spermatogenic cells DGCR8 localized not only to the nuclei but also to the cytoplasmic compartments, including nuage and nonnuage structures. Our IF results on the nuclear localization of DGCR8 are similar to those reported previously $[14,33]$. The staining intensity of DGCR8 


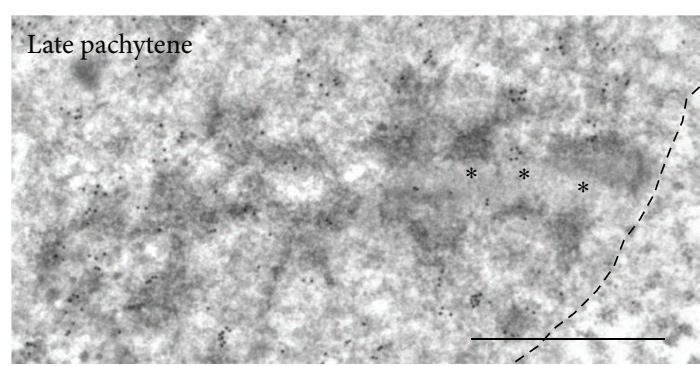

(a)

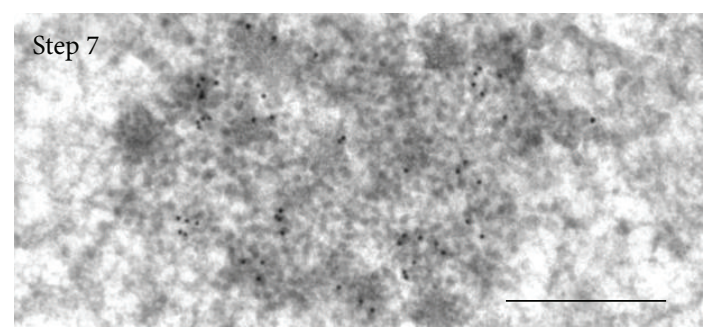

(b)

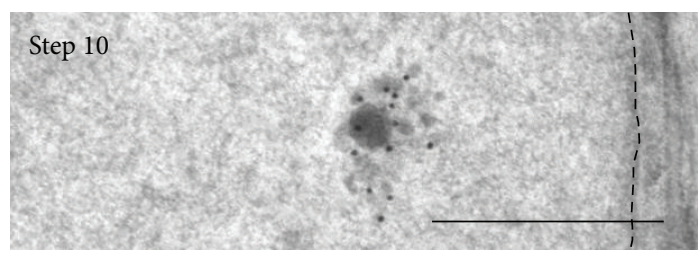

(c)

FIGURE 6: Localization of DGCR8 in the nuclei of spermatogenic cells by IEM. Data obtained with antibody from ProteinTech. Dashed line indicates nuclear envelope. (a) A nucleus of a late pachytene spermatocyte of mouse. Gold labeling is associated with euchromatin near heterochromatin but not with synaptonemmal complex $\left({ }^{*}\right)$. (b) Nucleoplasm of a step 7 spermatid of rat. An aggregate of small particles is labeled with gold particles but the other area not. (c) Nucleoplasm of a step 10 spermatid of rat. An aggregate of particles is labeled but the other area is not. Bar $=1 \mu \mathrm{m}$ for (a) and $0.5 \mu \mathrm{m}$ for (b) and (c).

in spermatogenic cells changed during differentiation of spermatogenic cells. In pachytene spermatocytes, the strongest staining was observed at around stage XIII. This suggests that the cells are preparing miRNA silencing pathway against transposon appearing in meiosis shortly after this stage [3436]. Our IEM study clarified that in the nucleolus DGCR8 was almost exclusively localized to granular component but not dense fibrillar component. The results are similar to previous report [14]. The nucleolus disappears in late spermatids. In such cells, DGCR8 was found to be associated with the aggregate of small dense particles in the nucleoplasm. The nature of these particles is unclear. Electron density of them is very similar to that of ribonucleoproteins.

4.3. DGCR8 in Nuage and Nonnuage Structures. Present study showed that in spermatogenic cells, DGCR8 localized

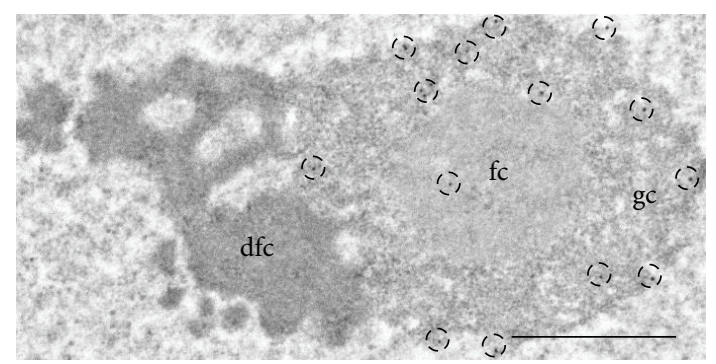

(a)

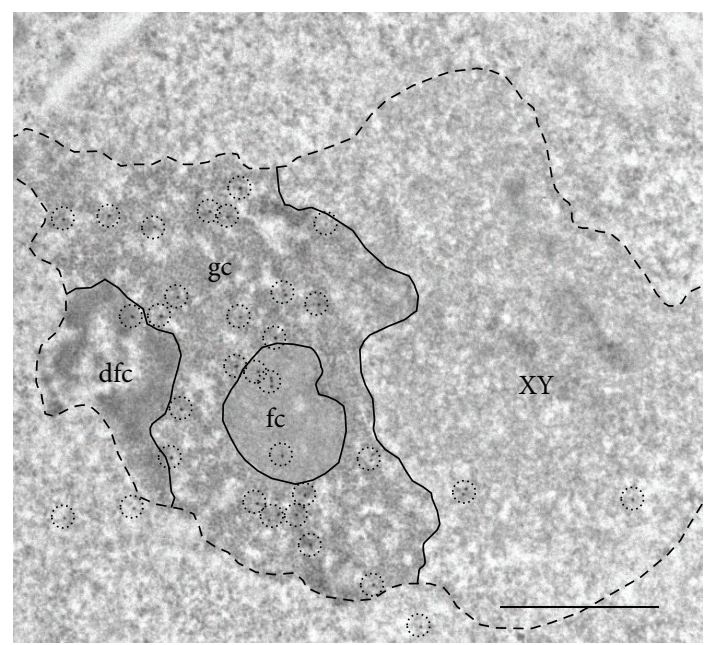

(b)

FIGURE 7: Localization DGCR8 in the nucleoli of pachytene spermatocytes by IEM. Data obtained with antibody from ProteinTech. Gold particles are marked by circles. (a) A nucleolus of a midspermatocyte. Gold particles are seen on granular component (gc) but a few or no on fibrillar component ( $\mathrm{fc}$ ) and dense fibrillar component (dfc). (b) A nucleolus associated with XY body. Gold labeling is observed on granular component (gc) and fibrillar component (fc) but not on dense fibrillar component ( $\mathrm{dfc}$ ). $\mathrm{XY}$ body $(\mathrm{XY})$ is weakly labeled. Bar $=1 \mu \mathrm{m}$.

to various cytoplasmic structures, including nuage and nonnuage structures specific for spermatogenic cells. The nuage is known to be a cytoplasmic site for RNA processing, including translation initiation [37, 38], RNA silencing [35, 39], RNA decay [40, 41], and RNA storage [23, 42]. The function of DGCR8 in nuage is not known. The localization of DGCR8 in the nuage suggests that it has some sort of function which is not relevant to serving as a subunit of microprocessor complex. In the present study, we showed that DGCR8 was present in nonnuage structures such as mitochondriaassociated granules, ribosome aggregates, and small granules in the residual bodies. We have revealed that several nuage proteins are also contained in these structures [25, 28, 29, 31], to which DGCR8 was added as a constituent protein in this time. However, the role of the nonnuage structures in spermatogenesis is quite unknown. This is our future problem to solve. 

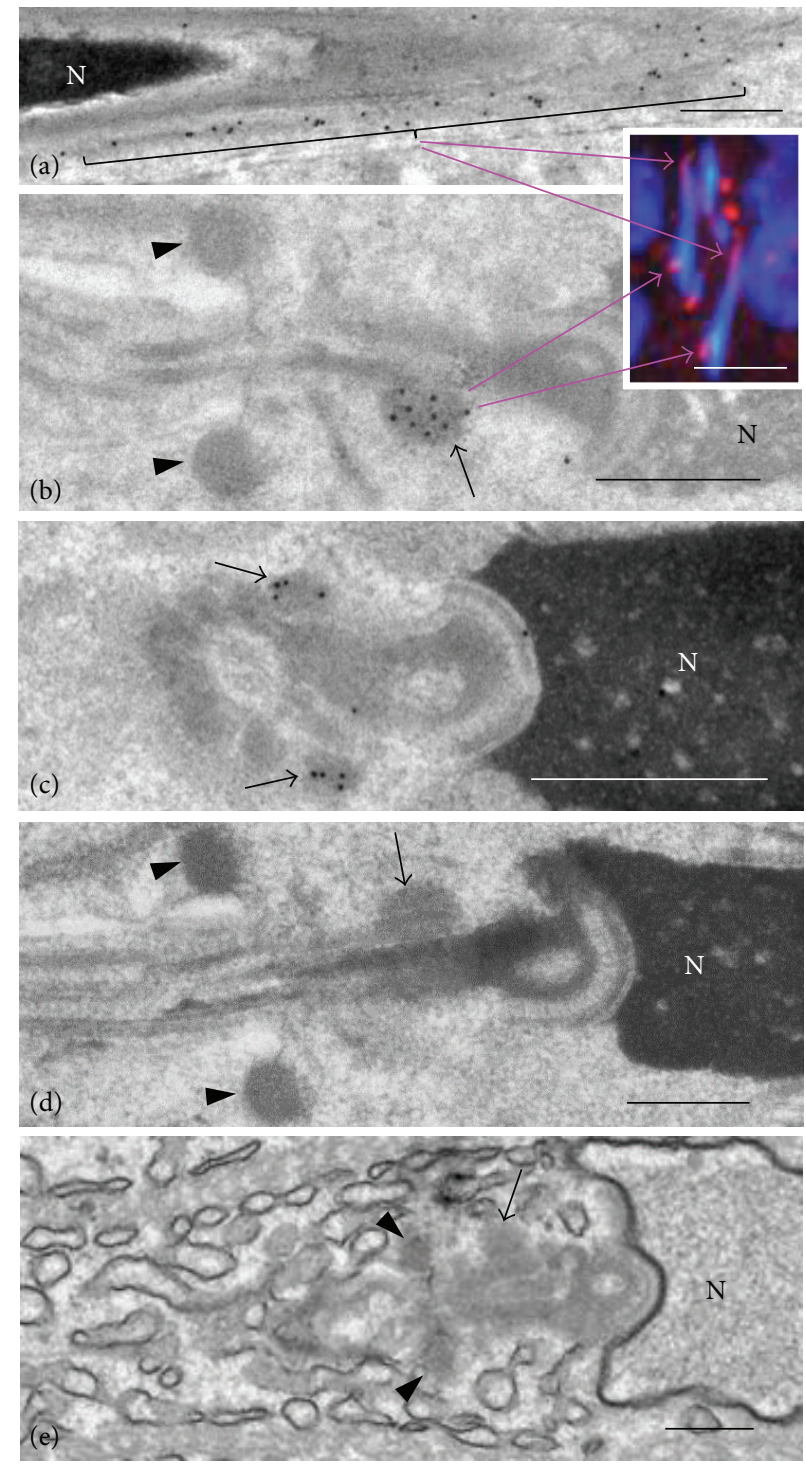

FIGURE 8: Localization of DGCR8 in the head and neck regions of rat late spermatids by IEM and the corresponding area stained by IF (inset). All data obtained with antibody from ProteinTech. (a) Apical area of a step 17 spermatid head. Gold particles are present on dense material in the cytoplasm of a Sertoli cell at the ectoplasmic specialization (bracket). The corresponding areas stained by IF are shown in inset (arrows). (b) A neck granule in a step 15 spermatid, which corresponds to granules stained by IF (Inset) (long arrows). Gold labeling is seen in the granule (short arrow) but not on cross-sectioned annulus (arrowheads). (c) Two neck granules are shown. They are positive for DGCR8 (arrows). (d) IEM control. No gold labeling is observed in a neck granule (arrow). Arrowheads indicate a cross-sectioned annulus. (e) Routine transmission EM image of neck region. A neck granule attaching to neck piece of flagellum is observed (arrow). A cross-sectioned annulus is pointed by arrowheads. N: nucleus. Bar $=0.5 \mu \mathrm{m}$

Interesting finding is the localization of DGCR8 in neck granules of late spermatids and epididymal sperm. In addition, the granules were confirmed to exist in sperm of pig and horse. The results suggest that DGCR8 is a constituent protein in the neck granules of mammals. Previously, we have shown that MAEL protein, which plays a central role as transposon silencer during spermatogenesis, localizes to neck granules [25]. The relationship between these proteins is not obvious. It is interesting that DGCR8 might be carried into egg by the neck granules during fertilization.

\section{Conclusion}

DGCR8 localizes not only to the nucleus but also to the cytoplasmic structures, including nuage and nonnuage structures in spermatogenic cells. In the nucleus, DGCR8 is mainly present in nucleolus, especially in the granular component. In the nuage, DGCR8 localizes to irregularly shaped perinuclear granules, intermitochondrial cement, and chromatoid bodies. DGCR8 localizes to the neck granules of late spermatids and epididymal sperm. 


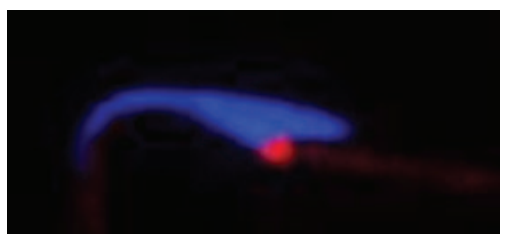

(a)

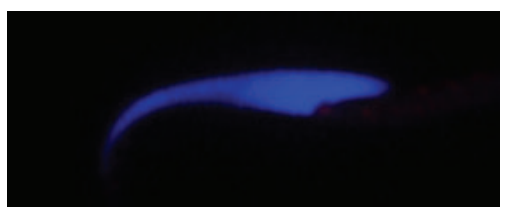

(b)

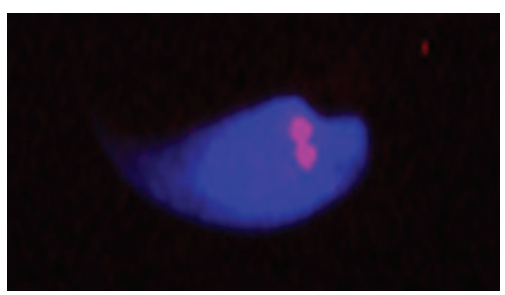

(c)

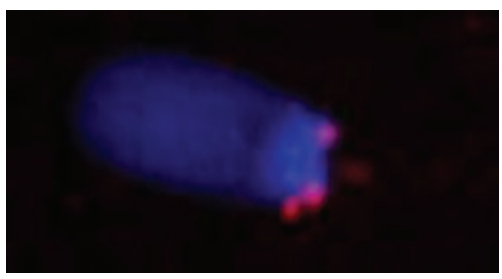

(d)

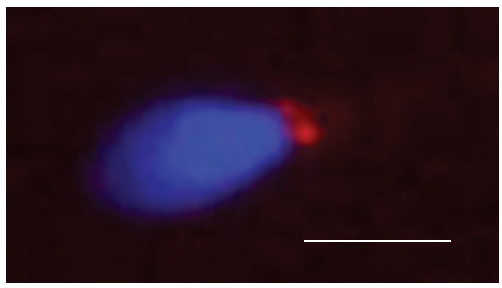

(e)

FIGURE 9: Localization of DGCR8 in epididymal sperm by IF. Data obtained by antibody from ProteinTech. (a) Rat. (b) IF control of rat. (c) Mouse. (d) Pig. (e) Horse. Bar $=5 \mu \mathrm{m}$.

\section{Acknowledgments}

The work was supported by the University Research Fund, in part by a Grant-in-aid (17570158) from the Ministry of Education, Science, Culture and Sport and by the Science Research Promotion Fund from the Promotion and Mutual Aid Corporation for Private Schools of Japan.

\section{References}

[1] E. M. Eddy, "Germ plasm and the differentiation of the germ cell line," International Review of Cytology, vol. 43, pp. 229-280, 1975.
[2] D. M. de Krester and J. B. Kerr, "The cytology of the testis," in The Physiology of Reproduction, E. Knobil and J. Neill, Eds., pp. 837-932, Raven Press, New York, NY, USA, 1988.

[3] Y. Clermont, R. Oko, and L. Hermo, "Cell biology of mammalian spermatogenesis," in Cell and Molecular Biology of the Testis, C. Desjardins and L. L. Ewing, Eds., pp. 332-376, Oxford University Press, New York, NY, USA, 1993.

[4] N. B. Hecht, "Gene expression during male germ cell development," in Cell and Molecular Biology of the Testis, C. Desjardins and L. L. Ewing, Eds., pp. 400-432, Oxford University Press, New York, NY, USA, 1993.

[5] M. Shoji, S. Chuma, K. Yoshida, T. Morita, and N. Nakatsuji, "RNA interference during spermatogenesis in mice," Developmental Biology, vol. 282, no. 2, pp. 524-534, 2005.

[6] K. Zheng, J. Xiol, M. Reuter et al., "Mouse MOV10L1 associates with Piwi proteins and is an essential component of the Piwiinteracting RNA (piRNA) pathway," Proceedings of the National Academy of Sciences of the United States of America, vol. 107, no. 26, pp. 11841-11846, 2010.

[7] R. J. A. Frost, F. K. Hamra, J. A. Richardson, X. Qi, R. BasselDuby, and E. N. Olson, "MOV10L1 is necessary for protection of spermatocytes against retrotransposons by Piwi-interacting RNAs," Proceedings of the National Academy of Sciences of the United States of America, vol. 107, no. 26, pp. 11847-11852, 2010.

[8] R. Song, G. W. Hennig, Q. Wu, C. Jose, H. Zheng, and W. Yan, "Male germ cells express abundant endogenous sirnas," Proceedings of the National Academy of Sciences of the United States of America, vol. 108, no. 32, pp. 13159-13164, 2011.

[9] J. Bao, D. Li, L. Wang et al., "MicroRNA-449 and microRNA$34 \mathrm{~b} / \mathrm{c}$ function redundantly in murine testes by targeting E2F transcription factor-retinoblastoma protein (E2F-pRb) pathway," Journal of Biological Chemistry, vol. 287, pp. 21686-21698, 2012.

[10] Y. Lee, C. Ahn, J. Han et al., "The nuclear RNase III Drosha initiates microRNA processing," Nature, vol. 425, no. 6956, pp. 415-419, 2003.

[11] A. M. Denli, B. B. J. Tops, R. H. A. Plasterk, R. F. Ketting, and G. J. Hannon, "Processing of primary microRNAs by the Microprocessor complex," Nature, vol. 432, no. 7014, pp. 231235, 2004.

[12] J. Han, Y. Lee, K.-H. Yeom, Y.-K. Kim, H. Jin, and V. N. Kim, "The Drosha-DGCR8 complex in primary microRNA processing," Genes and Development, vol. 18, no. 24, pp. 30163027, 2004.

[13] J. Winter, S. Jung, S. Keller, R. I. Gregory, and S. Diederichs, "Many roads to maturity: microRNA biogenesis pathways and their regulation," Nature Cell Biology, vol. 11, no. 3, pp. 228-234, 2009.

[14] A. Shiohama, T. Sasaki, S. Noda, S. Minoshima, and N. Shimizu, "Nucleolar localization of DGCR8 and identification of eleven DGCR8-associated proteins," Experimental Cell Research, vol. 313, no. 20, pp. 4196-4207, 2007.

[15] U. Sheth and R. Parker, "Decapping and decay of messenger RNA occur in cytoplasmic processing bodies," Science, vol. 300, no. 5620, pp. 805-808, 2003.

[16] J. Liu, M. A. Valencia-Sanchez, G. J. Hannon, and R. Parker, "MicroRNA-dependent localization of targeted mRNAs to mammalian P-bodies," Nature Cell Biology, vol. 7, no. 7, pp. 719723, 2005.

[17] N. Kedersha, G. Stoecklin, M. Ayodele et al., "Stress granules and processing bodies are dynamically linked sites of mRNP 
remodeling," Journal of Cell Biology, vol. 169, no. 6, pp. 871-884, 2005.

[18] P. Anderson and N. Kedersha, "RNA granules," Journal of Cell Biology, vol. 172, no. 6, pp. 803-808, 2006.

[19] R. Parker and U. Sheth, "P bodies and the control of mRNA translation and degradation," Molecular Cell, vol. 25, no. 5, pp. 635-646, 2007.

[20] N. Kotaja, S. N. Bhattacharyya, L. Jaskiewicz et al., "The chromatoid body of male germ cells: similarity with processing bodies and presence of Dicer and microRNA pathway components," Proceedings of the National Academy of Sciences of the United States of America, vol. 103, no. 8, pp. 2647-2652, 2006.

[21] N. Kotaja, H. Lin, M. Parvinen, and P. Sassone-Corsi, "Interplay of PIWI/Argonaute protein MIWI and kinesin KIF17b in chromatoid bodies of male germ cells," Journal of Cell Science, vol. 119, no. 13, pp. 2819-2825, 2006.

[22] N. Kotaja and P. Sassone-Corsi, "The chromatoid body: a germcell-specific RNA-processing centre," Nature Reviews Molecular Cell Biology, vol. 8, no. 1, pp. 85-90, 2007.

[23] E. Voronina, G. Seydoux, P. Sosson-Corsi, and I. Nagamori, "RNA granules in germ cells," Cold Spring Harbor Perspective in Biology, vol. 3, no. 12, Article ID a002774, 2011.

[24] S. S. Guraya, "Recent advances in the morphology, cytochemistry, and function of Balbiani's vitelline body in animal oocytes," International Review of Cytology, vol. 59, pp. 249-321, 1979.

[25] M. Takebe, Y. Onohara, and S. Yokota, "Expression of MAEL in Nuage and non-Nuage compartments of rat spermatogenic cells and colocalization with DDX4, DDX25 and MIWI," Histochemistry and Cell Biology, vol. 140, pp. 169-181, 2013.

[26] S. Yokota, "Preparation of colloidal gold particles and conjugation of protein $\mathrm{A}, \operatorname{IgG}, \mathrm{F}(\mathrm{ab})_{2}$, and streptavidin," in Immunoelectron Microscopy. Methods and Protocols, S. D. Schwarzbach and T. Osafune, Eds., pp. 109-119, Springer, New York, NY, USA edition, 2012.

[27] L. D. Russell, R. A. Ettlin, A. P. S. Hikim, and E. D. Clegg, Histological and Histopathological Evaluation of the Testis, Cache River Press, Clearwater, Fla, USA, 1990.

[28] Y. Onohara, T. Fujiwara, T. Yasukochi, M. Himeno, and S. Yokota, "Localization of mouse vasa homolog protein in chromatoid body and related nuage structures of mammalian spermatogenic cells during spermatogenesis," Histochemistry and Cell Biology, vol. 133, no. 6, pp. 627-639, 2010.

[29] Y. Onohara and S. Yokota, "Expression of DDX25 in nuage components of mammalian spermatogenic cells: immunofluorescence and immunoelectron microscopic study," Histochemistry and Cell Biology, vol. 137, no. 1, pp. 37-51, 2012.

[30] Y. Onohara and S. Yokota, "Nuage components and their contents in mammalian spermatogenic cells, as revealed by immunoelectron microscopy," in Meiosis-Molecular Mechanisms and Cytogenetic Diversity, A. Swan, Ed., pp. 217-240, Intech, 2012.

[31] S. Yokota, "Nuage proteins: their localization in subcellular structures of spermatogenic cells as revealed by immunoelectron microscopy," Histochemistry and Cell Biology, vol. 138, pp. 1-11, 2012.

[32] L. D. Russell, J. C. Goh, R. M. Rashed, and A. W. Vogl, "The consequences of actin disruption at Sertoli ectoplasmic specialization sites facing spermatids after in vivo exposure of rat testis to cytochalasin D," Biology of Reproduction, vol. 39, no. 1, pp. 105-118, 1988.
[33] K.-H. Yeom, Y. Lee, J. Han, M. R. Suh, and V. N. Kim, "Characterization of DGCR8/Pasha, the essential cofactor for Drosha in primary miRNA processing," Nucleic Acids Research, vol. 34, no. 16, pp. 4622-4629, 2006.

[34] M. A. Carmell, A. Girard, H. J. G. van de Kant et al., "MIWI2 is essential for spermatogenesis and repression of transposons in the mouse male germline," Developmental Cell, vol. 12, no. 4, pp. 503-514, 2007.

[35] S. F. C. Soper, G. W. van der Heijden, T. C. Hardiman et al., "Mouse maelstrom, a component of nuage, is essential for spermatogenesis and transposon repression in meiosis," Developmental Cell, vol. 15, no. 2, pp. 285-297, 2008.

[36] R. Öllinger, J. Reichmann, and I. R. Adams, "Meiosis and retrotransposon silencing during germ cell development in mice," Differentiation, vol. 79, no. 3, pp. 147-158, 2010.

[37] S. Chuma, M. Hiyoshi, A. Yamamoto, M. Hosokawa, K. Takamune, and N. Nakatsuji, "Mouse Tudor Repeat-1 (MTR1) is a novel component of chromatoid bodies/nuages in male germ cells and forms a complex with snRNPs," Mechanisms of Development, vol. 120, no. 9, pp. 979-990, 2003.

[38] T. V. Pestova, V. G. Kolupaeva, I. B. Lomakin et al., "Molecular mechanisms of translation initiation in eukaryotes," Proceedings of the National Academy of Sciences of the United States of America, vol. 98, no. 13, pp. 7029-7036, 2001.

[39] Y. Yabuta, H. Ohta, T. Abe, K. Kurimoto, S. Chuma, and M. Saitou, "TDRD5 is required for retrotransposon silencing, chromatoid body assembly, and spermiogenesis in mice," Journal of Cell Biology, vol. 192, no. 5, pp. 781-795, 2011.

[40] J. M. Coller, M. Tucker, U. Sheth, M. A. Valencia-Sanchez, and R. Parker, "The DEAD box helicase, Dhhlp, functions in mRNA decapping and interacts with both the decapping and deadenylase complexes," RNA, vol. 7, no. 12, pp. 1717-1727, 2001.

[41] N. Minshall, G. Thom, and N. Standart, "A conserved role of a DEAD box helicase in mRNA masking," $R N A$, vol. 7, no. 12, pp. 1728-1742, 2001.

[42] A. Vasileva, D. Tiedau, A. Firooznia, T. Müller-Reichert, and R. Jessberger, "Tdrd6 is required for spermiogenesis, chromatoid body architecture, and regulation of miRNA expression," Current Biology, vol. 19, no. 8, pp. 630-639, 2009. 

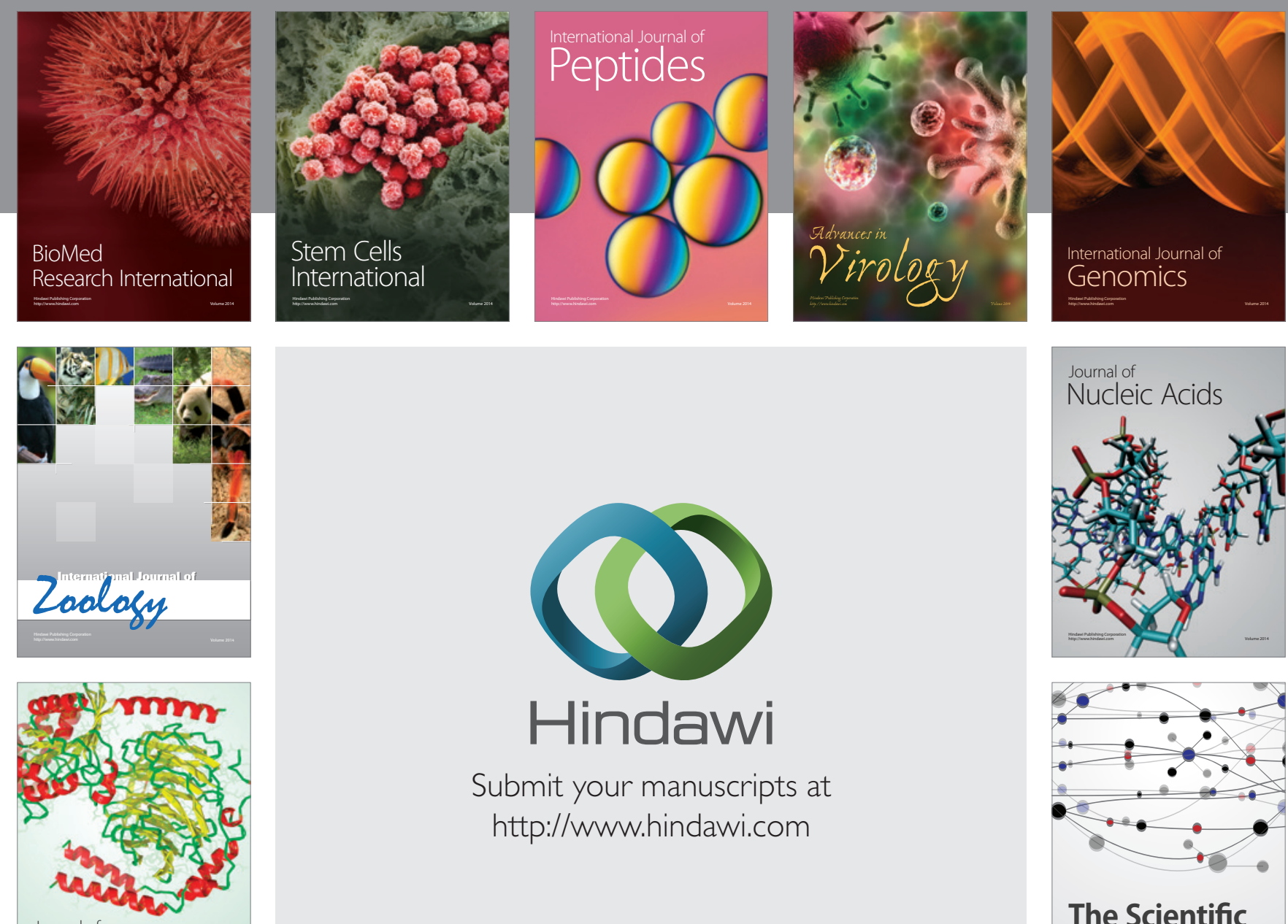

Submit your manuscripts at

http://www.hindawi.com

Journal of
Signal Transduction
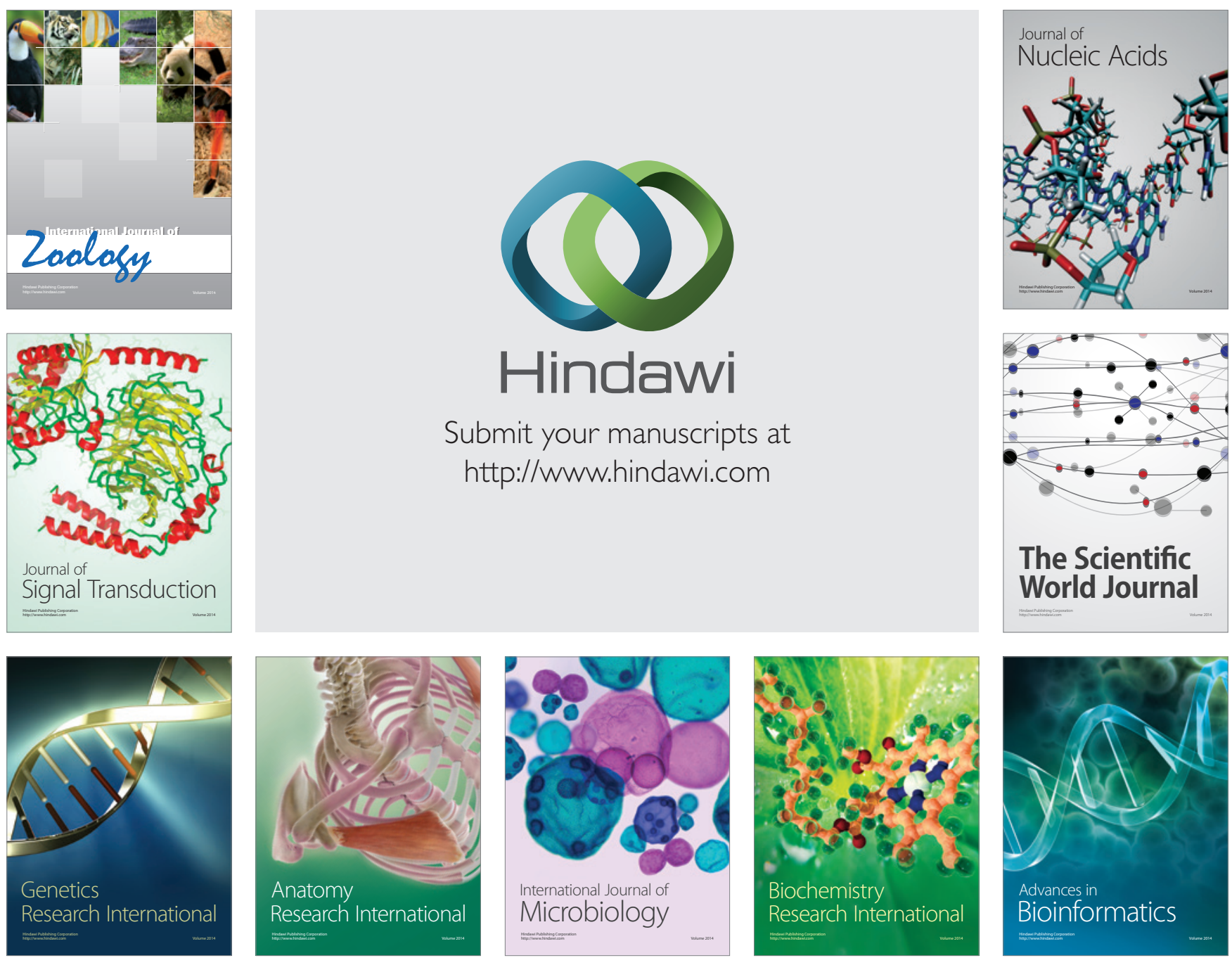

The Scientific World Journal
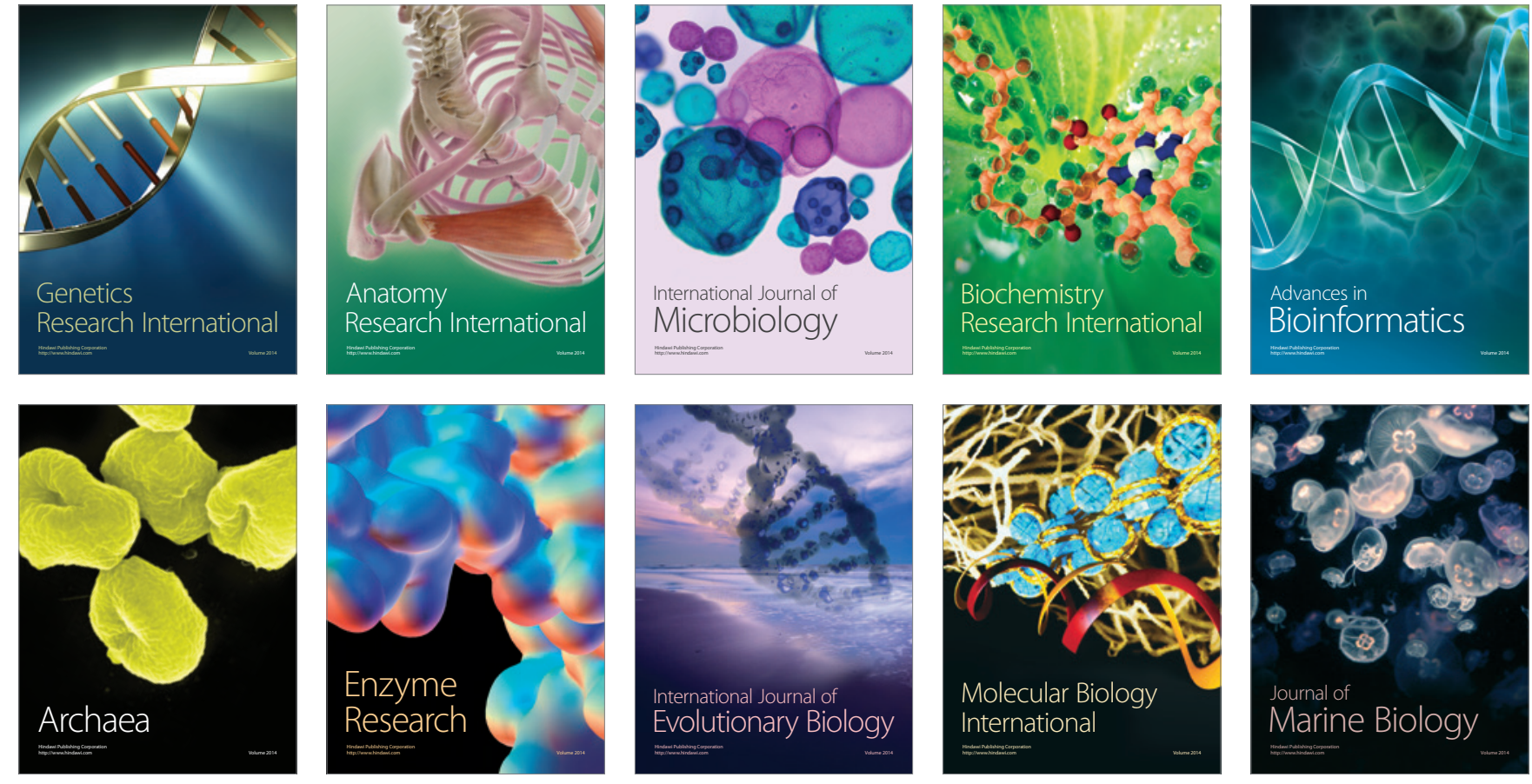